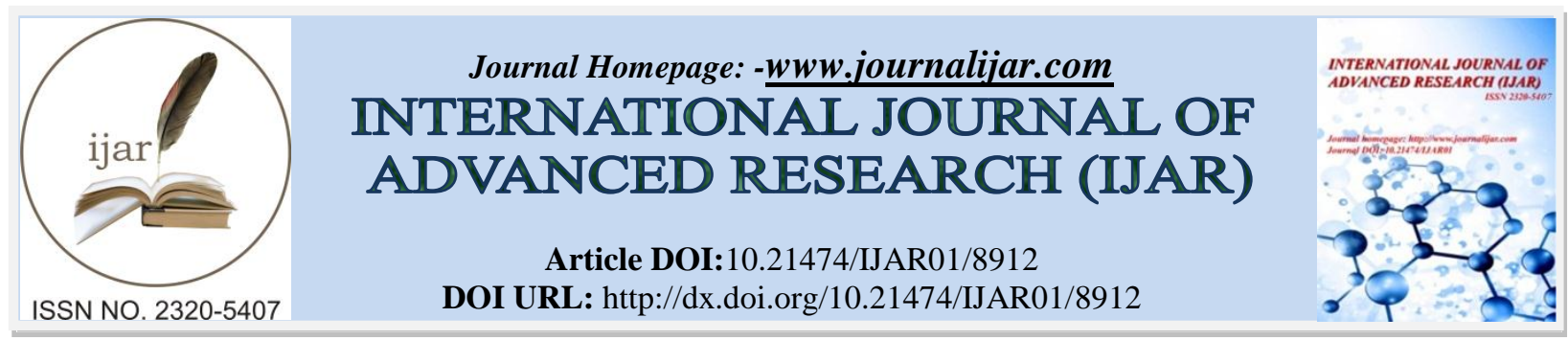

RESEARCH ARTICLE

\title{
DESIGNING AN IMPROVED RESISTIVE SENSOR INTERFACE WITH CNFET.
}

Farrukh Arslan.

School of Electrical and Computer Engineering, Purdue University, West Lafayette, IN, USA.

\section{Manuscript Info}

\section{Manuscript History}

Received: 12 February 2019

Final Accepted: 14 March 2019

Published: April 2019

\section{Key words:-}

Carbon nanotube (CNT), Carbon nanotube field effect transistor (CNFET), Differential Voltage Current Conveyor (DVCC), Resistive Sensor Interface.

\begin{abstract}
Sensor interface has been widely used in applications areas such as pharmacy, food-processing, biotechnology, industries, and laboratories etc. The differential voltage current conveyor (DVCC) is a very versatile active building block and attained specialinterest for currentmode circuits. This paper attempts to explore an application of CNFETbased current conveyor (DVCC) for the design of a resistive sensor interface. The proposed interface circuit reduces $16.6 \%$ power than existing work. The design also minimizes the number of active and passive components in the circuit. Further, a DVCC based Instrumentation amplifier is presented which is also utilized for the simpler design of sensor interface. HSPICE simulations with $32 \mathrm{~nm}$ CNFET model are performed to test the design.
\end{abstract}

Copy Right, IJAR, 2019,. All rights reserved.

\section{Introduction:-}

Since the last few years, there is a trend to grow integrated sensors on silicon substrate because of their compatibility with CMOS technology. These systems have the advantages like improved sensitivity, small size, low power and cost effectiveness [11]. Although, before the development of microelectronics, the sensors or transducers are used to measure physical quantities, such as temperature, pressure, and flow. That is coupled directly to a readout device from which a person can take the readings. In this system, the transducer converts the physical quantity into a displacement in the scale. However, a sensor with its interface circuitry has many benefits. The circuit designer can trade off useless performance characteristics for those that will provide improved performance to the sensorinterface circuit $[6,20,22]$. From the last thirty years, the electronics industry has undergone a significant scaling of transistor sizes with the number of components on an IC approximately increasing double in every 18-24 months. However, as the size of transistor scaled below $10 \mathrm{~nm}$, short channel effects and source (S)-Drain (D) tunneling arise $[10,15]$. Thus, the industry and engineers are looking for new substitute materials and devices to combine with current CMOS process technology [14, 19].

The carbon nano tube field effect transistor (CNFET) is an emerging technology which can be a possible alternative to solve the limitations of CMOS technology.

The CNFET is similar to MOS transistor, except the channel region contains carbon nanotubes (CNTs). The CNFET has outstanding properties like higher drive current, higher carrier mobility, higher transconductance and good control over the channel, as compared to its CMOS counterpart [4, 5, 25].

Corresponding Author:-Farrukh Arslan.

Address:-School of Electrical and Computer Engineering, Purdue University, West Lafayette, IN, USA. 
In this work, a comparison between CMOS and CNFET based DVCC is presented. Moreover, an improved CNFET based resistive sensor interface is proposed which reduces the active \& passive components and power requirement than the existing work [9]. Further, the work also attempts to investigate the use of CNFET based Instrumentation amplifier (IA) for a simpler interface design. This rest of the paper is prepared in the following manner. The implementation of the differential voltage current conveyor (DVCC) using carbon nanotube-FETs is discussed in Section 2. Section 3 presents the improved circuit of the resistive sensor interface. Section 4 deals with simulation results and discussion. Section 5 presents the conclusion.

\section{Brief Discussion on DVCC}

The current conveyor was introduced by Sedra\& Smith and after that many versions of current conveyors were discussed [1,3] but the second generation current conveyor (CC-II) proved to a versatile active element for the realization of current-mode (CM) and voltage-mode (VM) circuits. The CC-II is not suitable for differential input signals, as it has the one high impedance node (Y). However, the differential voltage current conveyor (DVCC) filled this gap, as DVCC has an extra Y terminal to manage differential inputs. The symbol of DVCC has been depicted in Fig. 1. The input-output ports relation is given by (1).

$$
I_{Y 1}=I_{Y 2}=0, I_{Z} \pm= \pm I_{X}, V_{X}=V_{Y 1}-V_{Y 2}
$$

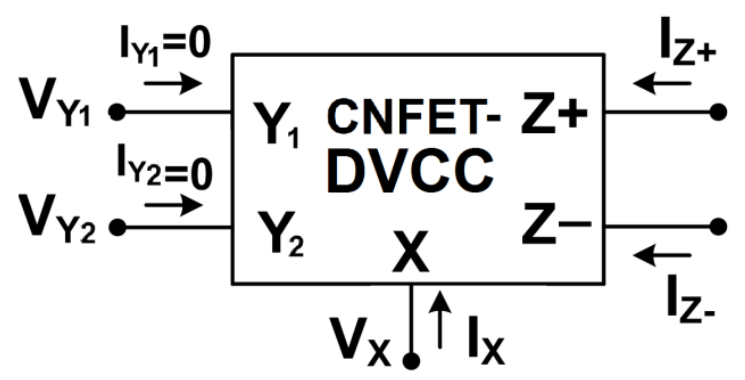

Figure 1:-Circuit Symbol of DVCC [12]

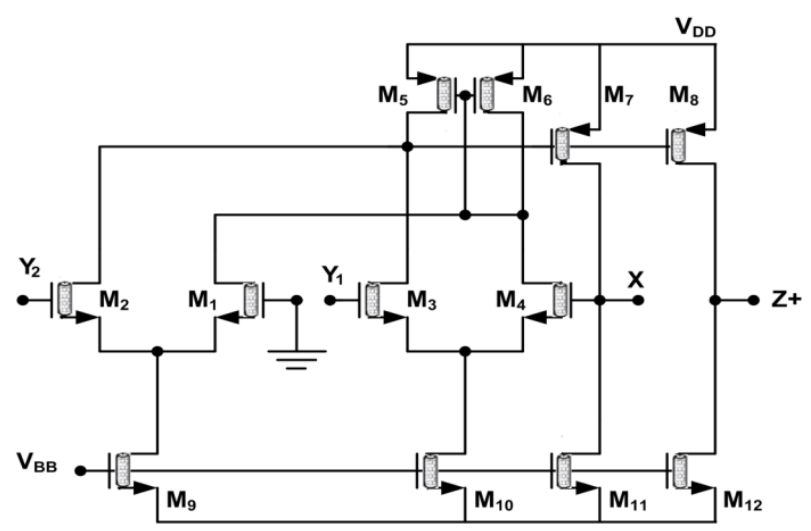

Figure 2:-Transistor Level Implementation of DVCC with Z+ node only

The transistor level implementation of DVCC based on CNFET is illustrated and given in Fig. 2. Here, HSPICE 32 nm CNFET parameters are used for the design which is developed by Stanford University [7]. The design parameters of CNFET are presented in Table 1.

Table 1:-CNFET Parameters Considered for DVCC

\begin{tabular}{|l|l|}
\hline Parameters & Value \\
\hline Oxide Thickness $\left(\mathrm{T}_{\mathrm{OX}}\right)$ & $4 \mathrm{~nm}$ \\
\hline Dielectric Constant $\left(\mathrm{K}_{\mathrm{OX}}\right)$ & 16 \\
\hline Power Supply & $0.75 \mathrm{~V}$ \\
\hline
\end{tabular}




\begin{tabular}{|l|l|}
\hline Chirality of the tube $(\mathrm{n}, \mathrm{m})$ & 19,0 \\
\hline Physical channel length $\left(\mathrm{L}_{\mathrm{ch}}\right)$ & $32 \mathrm{~nm}$ \\
\hline No. of tubes in the device & 6 \\
\hline Pitch $(\mathrm{S})$ & $14 \mathrm{~nm}$ \\
\hline
\end{tabular}

Considering the circuit of Fig. 2, basic operation can be explained as follows. The transistorsM5 andM6, work as a current mirror which is set to drive two differential amplifiers consisting of transistors $M_{1} \& M_{2}$ and $M 3 \& M_{4}$. Additionally, the sum of drain currents of $\mathrm{M}_{1}$ and $\mathrm{M}_{4}$ is equal to drain currents of $\mathrm{M}_{2}$ and $\mathrm{M}_{3}$. Further, since transistors $\mathrm{M}_{9}$ and $\mathrm{M}_{10}$ are biased with equal gate voltages (and since their source voltages are also equal), they would have equal drain currents. The relation between $\mathrm{X}$ and $\mathrm{Y}$ nodes presented as (2). Additionally, transistors $\mathrm{M}_{7}$ andM $M_{11}$ provide the necessary feedback action to make the voltage VX independent of current drawn from the terminal $\mathrm{X}$. The current in terminal $\mathrm{X}$ is conveyed to the $\mathrm{Z}+$ terminal with the help of transistors $\mathrm{M}_{7}, \mathrm{M}_{8}, \mathrm{M}_{11}$ and $\mathrm{M}_{12}$.

$\mathrm{V}_{\mathrm{X}}=\mathrm{V}_{\mathrm{Y} 1}-\mathrm{V}_{\mathrm{Y} 2}$

\section{Overview of Nanotube Transistor}

The carbon nanotube (CNT) has introduced as a key component for present nano-scale circuit design. Apart from their extensive use in chemistry, biotechnology, material science etc., CNTs have also outperformed over the conventional MOSFETs for high performance and low power circuits, as carbon nanotube electronics offers higher current carrying capability, higher transconductance and near ballistic transport of charge carriers [8, 13]. Carbon Nanotube Field Effect Transistors (CNFETs), have semiconducting single-walled Carbon Nanotubes (CNTs) as a channel material, show enormous potential as extensions to the silicon MOSFETs [16, 21, 24, 27]. A simplified structure of CNFET is shown in Fig. 3. Here, carbon nanotubes work as channel material for the MOSFET-like device.

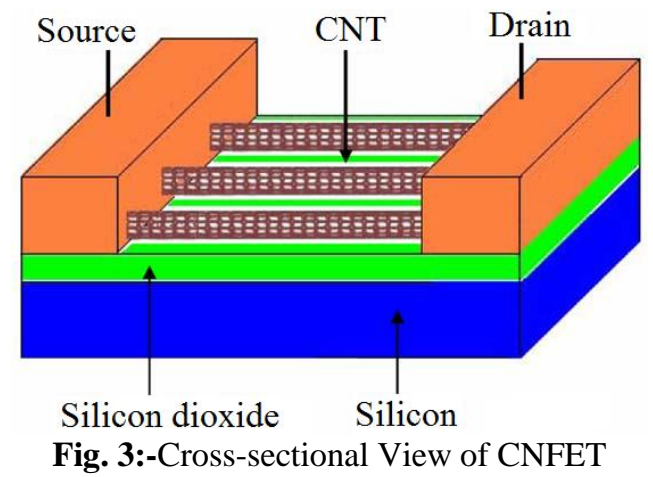

\section{Design of Improved Sensor Interface using DVCC}

The circuit of Fig. 4 was proposed by Giuseppe Ferriet. al. [9]. The circuit utilizes second generation current conveyor (CC-II) as an active element. It has three active and four passive components. Moreover, the number of active and passive components can be reduced by using differential voltage current conveyor (DVCC).



Fig 4:-Existing Resistive Interface Circuit [9] 
Fig. 5 shows the proposed circuit of the sensor interface. The proposed circuit uses two active and three passive components. The resistive sensor is placed as $R_{S}$. The routine analysis of the circuit is expressed by (1)-(8).

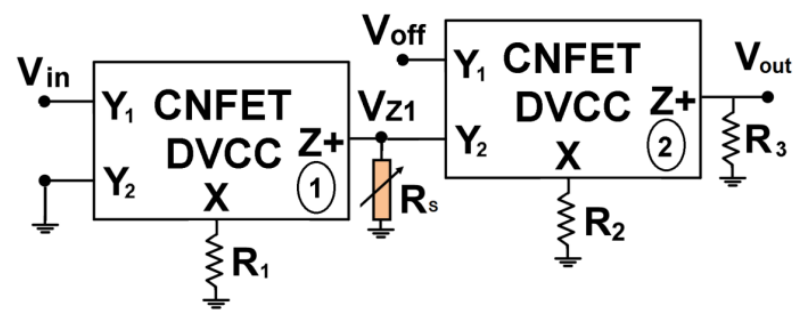

Fig 5:-Proposed Resistive Sensor Interface

$$
\begin{aligned}
& V_{X 1}=V_{i n} \\
& I_{X 1}=\frac{V_{\text {in }}}{R_{1}} \\
& V_{Z 1}=\frac{V_{\text {in }}}{R_{1}} * R_{S} \\
& V_{X 2}=V_{\text {off }}-V_{Z 1} \\
& I_{X 2}=\frac{V_{o f f}-V_{Z 1}}{R_{2}}=I_{Z 2}
\end{aligned}
$$

The variation in sensor's resistance $\left(\mathrm{R}_{\mathrm{S}}\right)$ is rejected in the output voltage of first DVCC (3). Thus, voltage $\left(\mathrm{V}_{\mathrm{X} 2}\right)$ of second DVCC is also change (4), which finally alter the output voltage $\left(\mathrm{V}_{\text {out }}\right)$ according to change in the resistance of sensor (8). Therefore, variation in sensor's resistance is measured by the change in the output voltage of proposed sensor interface.

$$
\begin{aligned}
& V_{\text {out }}=I_{Z 2} R_{3}=\frac{R_{3}}{R_{2}}\left[V_{\text {off }}-V_{Z 1}\right] \\
& V_{\text {out }}=\frac{R_{3}}{R_{2}}\left[V_{\text {off }}-V_{\text {in }}\left(R_{S} / R_{1}\right)\right] \\
& V_{\text {out }}=\frac{R_{3}}{R_{1} R_{2}}\left[V_{\text {off }} R_{1}-V_{\text {in }} R_{S}\right]
\end{aligned}
$$

Table 2:-Comparison of the Interface Circuits

\begin{tabular}{|l|l|l|}
\hline Parameter & {$[\mathbf{9 ]}$} & Proposed Work \\
\hline Active Elements & 3 & 2 \\
\hline Passive Elements & 4 & 3 \\
\hline VDD & $1.5 \mathrm{~V}$ & $0.75 \mathrm{~V}$ \\
\hline Power Consumption & $210 \mu \mathrm{W}$ & \\
\hline
\end{tabular}

In addition to this, it is important to compare the parameters of existing and proposed circuits of the resistive sensor interface. Table 2 presents the fair comparison between these two circuit designs.

\section{Resistive Sensor Interface using Instrumentation Amplifier}

In the last section, an improved circuit of sensor interface is discussed. But, there is a simpler design proposed for resistive sensor interface. The feature of DVCC can also be utilized as an Instrumentation Amplifier (IA) [12]. That 
concept can be further proposed as a circuit for sensor interface as shown in Fig. 6. It utilizes a DVCC and a resistor with resistive sensor $\left(\mathrm{R}_{\mathrm{S}}\right)$.

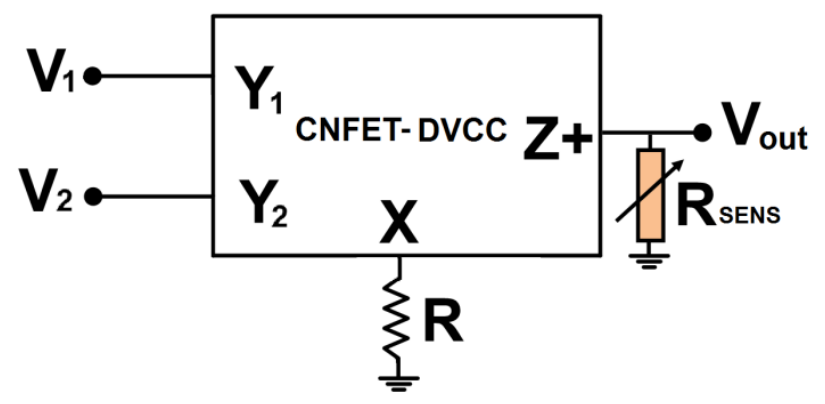

Fig 6:-Resistive Sensor Interface using IA

$V_{X}=V_{1}-V_{2}$

$I_{X}=\frac{V_{1}-V_{2}}{R_{1}}=I_{Z}$

$V_{\text {out }}=I_{Z} R_{2}=\frac{R_{2}}{R_{1}}\left(V_{1}-V_{2}\right)$

The relation (9) and (10) can be understood by properties of DVCC [18]. Moreover, change in (RS) is incorporated by the output voltage (11).

\section{Simulation Results:-}

The performance of CNFET-based DVCC with $32 \mathrm{~nm}$ feature size of CNFET is presented. The differential voltage current conveyor is simulated and tested through HSPICE, satisfying the characteristics as depicted in (1). Further, the current and voltage transfer characteristics of proposed building block are also tested with the fundamental properties. Fig. 7 and Fig. 8 illustrate the current and voltage transfer curves of the CNFET-based current conveyor. The input and output voltages have the linear relation i.e. $\mathrm{V}_{\mathrm{X}}=\left(\mathrm{V}_{\mathrm{Y} 11}-\mathrm{V}_{\mathrm{Y}_{2}}\right)$.

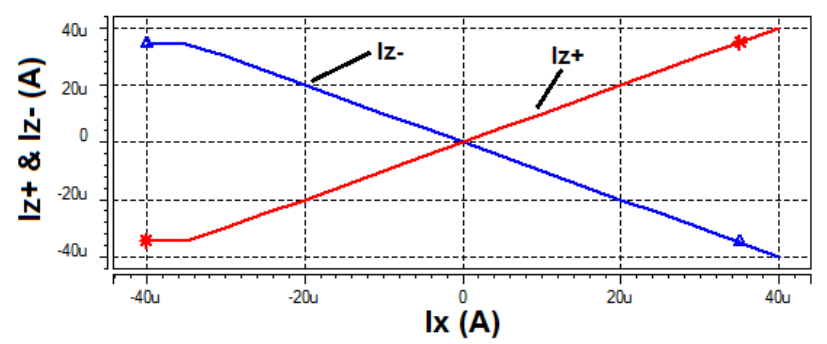

Fig 7:-DC Current Transfer Characteristics of CNFET-based DVCC

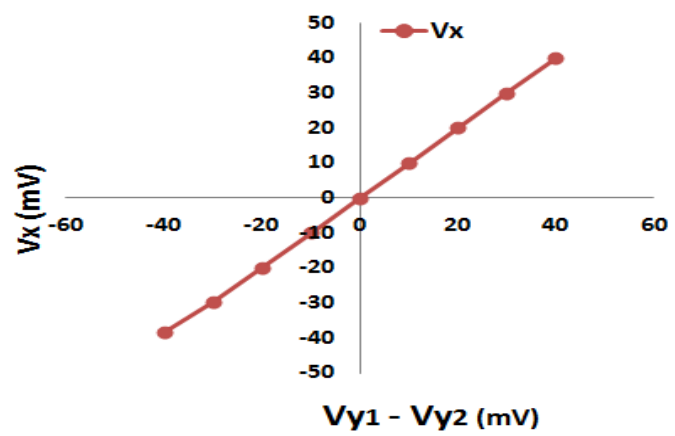

Fig 8:-DC Voltage Transfer Characteristics of CNFET-based DVCC 
Additionally, to test the validity of DVCC circuit, transient-mode simulations are also performed. The response of input-output voltages is given in Fig. 9, while

Fig. 10 reflects the currents of $\mathrm{X}$ and $\mathrm{Z}$ terminals of DVCC, as given in relations described by (1).

The comparison of CMOS and CNFET based DVCC is also performed. Table 3 illustrates the superiority of CNFET based DVCC over its CMOS counterpart. The CNFET based DVCC has 3.26 times improvement in bandwidth and 5.16 times reduction in power consumption than CMOS.

Table 3:-Comparison of CMOS and CNFET-based DVCC

\begin{tabular}{|l|l|l|}
\hline \multirow{2}{*}{ Parameter } & DVCC & \multicolumn{2}{|l|}{} \\
\cline { 2 - 3 } & CMOS & CNFET \\
\hline Model/Technology & PTM 32 nm & Stanford 32 nm \\
\hline Supply Voltage & $0.75 \mathrm{~V}$ & $0.75 \mathrm{~V}$ \\
\hline No. of Transistor & 12 & 12 \\
\hline 3-dB Bandwidth & $4.21 \mathrm{GHz}$ & $23.15 \mathrm{GHz}$ \\
\hline Power Consumption & $794.1 \mu \mathrm{W}$ & $110.41 \mu \mathrm{W}$ \\
\hline
\end{tabular}

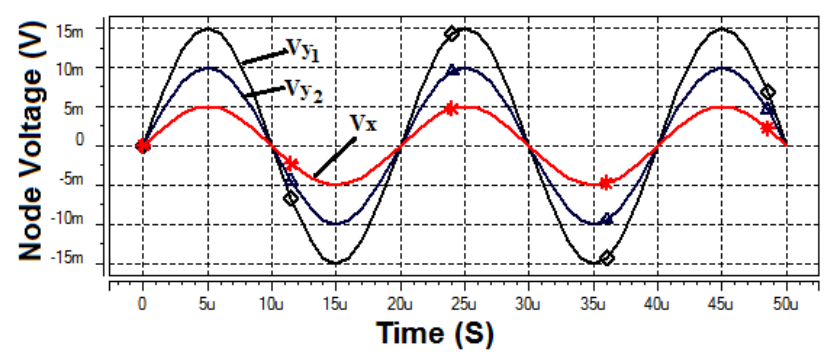

Fig 9:-Transient Voltage Characteristics of $\mathrm{V}_{\mathrm{X}}, \mathrm{V}_{\mathrm{Y} 1}$ and $\mathrm{V}_{\mathrm{Y} 2}$

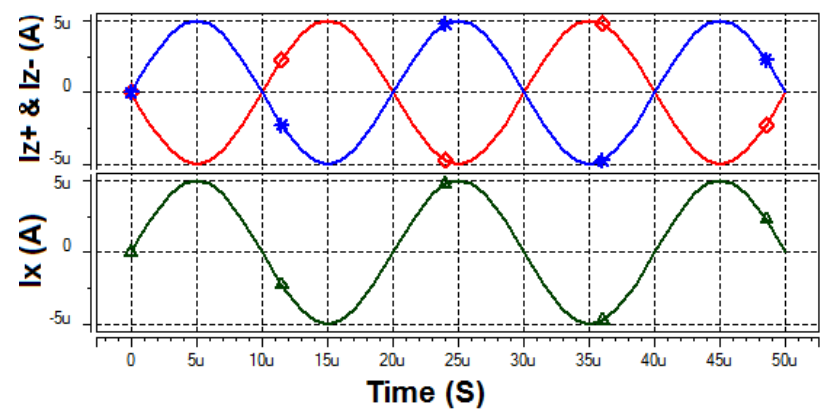

Fig 10:-Transient Current Characteristics of $\mathrm{I}_{\mathrm{X}}, \mathrm{I}_{\mathrm{Z}+}$ and $\mathrm{I}_{\mathrm{Z} \text { - }}$

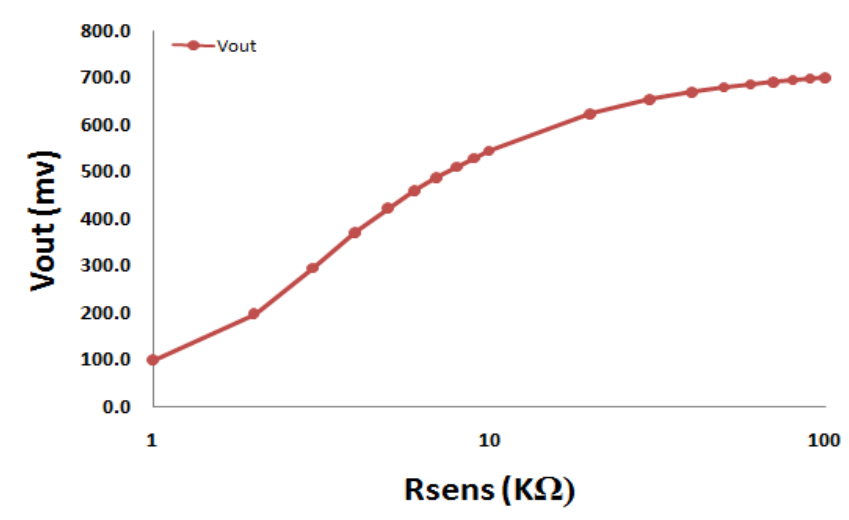


Fig 11:-Simulation Result of Resistive Sensor Interface

The sensor interface circuit using IA is realized with CNFET based DVCC. The circuit of Fig. 6 is simulated for the resistance range of 1-100 K. The corresponding output voltage is depicted in Fig. 11. Thus, CNFET based design can also be used in the circuits where low power electronic interface is required.

\section{Conclusion:-}

This work has explored the design of low-voltage low-power resistive sensor interface circuit. The proposed circuit used CNFET-based active building block for the interfacing of resistive sensor. The power requirement of the circuit is about $175 \mu \mathrm{W}$ with the supply voltage of $0.75 \mathrm{~V}$. Further; the results showed that the proposed circuit has the advantage over existing circuit as it utilizes only two active and three passive components. The sensor interface circuit could be used in various research and industrial applications. The work also presented the comparison between CMOS and CNFET based DVCC. The CNFET based current conveyor has 5.16-and 3.26-times improvement in power requirement and 3-dB bandwidth respectively than its CMOS counterpart. It shows that CNFET gives superior performance than CMOS. The circuit performed HSPICE simulations with $32 \mathrm{~nm}$ model to test the functionality.

\section{References:-}

1. M. T. Abuelmaatti and N. Almutairi. New current-feedback operational amplifier based bandpass shadow filter. International Journal of Electrical Engineering Education, 54(1):95-101, 2017.

2. A. Aouaj, A. Bouziane, and A. Nouacry. Nanotube carbon transistor (cntfet): $\mathrm{I}-\mathrm{V}$ and $\mathrm{C}-\mathrm{V}$ a qualitative comparison between fettoy simulator and compact model. In Multimedia Computing and Systems, 2009. ICMCS'09. International Conference on,

3. pages 236-239. IEEE, 2009.

4. D. Biolek, R. Senani, V. Biolkova, and Z. Kolka. Active elements for analog signal processing: classi_cation, review, and new proposals. Radioengineering, 17(4):15-32, 2008.

5. R. Bogue. The fabrication and assembly of nanoelectronic devices. Assembly Automation, Emerald, 30(3):206$212,2010$.

6. 5. R. J. Cobley. Book review: Nanotechnology for telecommunications, 2011.

7. 6. A. De Marcellis and G. Ferri. Analog circuits and systems for voltage-mode and current-mode sensor interfacing applications. Springer, 2011.

8. 7. J. Deng and H.-S.Wong. A compact spice model for carbon-nanotube Field-effect transistors including nonidealities and its applicationpart ii: Full device model and circuit performance benchmarking. Electron Devices, IEEE Transactions on, 54(12):3195- 3205, 2007.

9. 8. M. Dresselhaus, G. Dresselhaus, J.-C. Charlier, and E. Hernandez. Electronic, thermal and mechanical properties of carbon nanotubes. Philosophical Transactions of the Royal Society of London. Series A: Mathematical, Physical and Engineering Sciences, 362(1823):2065-2098, 2004.

10. 9. G. Ferri, V. Stornelli, and M. Fragnoli. An integrated improved ccii topology for resistive sensor application. Analog Integrated Circuits and Signal Processing, 48(3):247- 250, 2006.

11. 10. A. D. Franklin, M. Luisier, S.-J. Han, G. Tulevski, C. M. Breslin, L. Gignac, M. S. Lundstrom, and W. Haensch. Sub-10 nm carbon nanotube transistor. Nano letters, 12(2):758-762, 2012.

12. 11. W. Haensch, E. J. Nowak, R. H. Dennard, P. M. Solomon, A. Bryant, O. H. Dokumaci, A. Kumar, X. Wang, J. B. Johnson, and M. V. Fischetti. Silicon cmos devices beyond scaling. IBM Journal of Research and Development, 50(4.5):339-361, 2006.

13. 12. T. M. Hassan and S. A. Mahmoud. New cmos dvcc realization and applications to instrumentation amplifier and active-rc filters. AEU-International Journal of Electronics and Communications, 64(1):47-55, 2010.

14. 13. A. Jorio, G. Dresselhaus, and M. S. Dresselhaus. Carbon nanotubes: advanced topics in the synthesis, structure, properties and applications, volume 111. Springer, 2007.

15. 14. Y.-B. Kim. Challenges for nanoscale mosfets and emerging nanoelectronics. transac-tions on electrical and electronic materials, 11(3):93-105, 2010.

16. 15. K. J. Kuhn. Considerations for ultimate cmos scaling. IEEE Trans. Electron Devices, 59(7):1813-1828, 2012.

17. 16. Y.-M. Lin, J. Appenzeller, Z. Chen, and P. Avouris. Electrical transport and 1/f noise in semiconducting carbon nanotubes. Physica E: Low-dimensional Systems and Nanostructures, 37(1):72-77, 2007. 
18. 17. S. Masoumi, S. Masoumi, H. Hajghassem, H. Hajghassem, A. Erfanian, A. Erfanian, A. Molaei Rad, and A. Molaei Rad. Design and manufacture of tht explosives detector sensors based on cntfet. Sensor Review, Emerald, 36(4):414-420, 2016.

19. 18. S. Minaei and E. Yuce. All-grounded passive elements voltage-mode dvcc-based universal filters. Circuits, Systems and Signal Processing, 29(2):295-309, 2010.

20. 19. A. Musatov, Y. V. Gulyaev, K. Izraelyants, E. Kukovitskii, N. Kiselev, O. Y. Maslennikov, I. Guzilov, O. Zhigalina, A. Ormont, and E. Chirkova. Low-voltage planar field emitters based on carbon nanotubes. Journal of Communications Technology and Electronics, 51(8):960-964, 2006.

21. 20. S. Nihtianov and A. Luque. Smart sensors and mems: intelligent devices and microsystems for industrial applications. Woodhead Publishing, 2014.

22. 21. N. Patil, A. Lin, E. R. Myers, K. Ryu, A. Badmaev, C. Zhou, H.-S. Wong, and S. Mitra. Wafer-scale growth and transfer of aligned single-walled carbon nanotubes. Nanotechnology, IEEE Transactions on, 8(4):498-504, 2009.

23. 22. M. Przybylski. Data acquisition for sensor systems. International Journal of Electrical Engineering Education, 35(1):95, 1998.

24. A. Raychowdhury, S. Mukhopadhyay, and K. Roy. A circuit-compatible model of ballistic carbon nanotube field-effect transistors. Computer-Aided Design of Integrated Circuits and Systems, IEEE Transactions on, 23(10):1411-1420, 2004.

25. 24. A. Raychowdhury and K. Roy. Carbon nanotube electronics: design of high-performance and low-power digital circuits. IEEE Transactions on Circuits and Systems I: Regular Papers, 54(11):2391-2401, 2007.

26. 25. S. K. Tripathi and M. S. Ansari. Voltage-mode universal filter for Zigbee using $\pm 0.9 \mathrm{~V} 32 \mathrm{~nm}$ CNFET ICCII. 5th International Conference- (Conuence-2014), pp. 471-475 IEEE, 2014.

27. 26. S. K. Tripathi, M. S. Ansari, and A. M. Joshi. Low-noise tunable band-pass filter for ism 2.4 ghz bluetooth transceiver in $\pm 0.7 \mathrm{~V} 32 \mathrm{~nm}$ cnfet technology. In Proceedings of the International Conference on Data Engineering and Communication Technology, pages 435-443. Springer, 2017.

28. 27. S. Wind, J. Appenzeller, R. Martel, V. Derycke, and P. Avouris. Fabrication and electrical characterization of top gate single-wall carbon nanotube field-effect transistors. Journal of Vacuum Science \& Technology B, 20(6):2798-2801, 2002. 